\title{
Modelo Teórico de Sensor basado en el Principio de la Reflectometría de Luz sobre Fibra Óptica para Detección de Sobrecarga Vehicular
}

\author{
Hernán Paz-Penagos ${ }^{*}$, Sandra X. Campagnoli-Martínez, Juan C. Gómez-Rubiano y Juan S. Currea. \\ Escuela Colombiana de Ingeniería "Julio Garavito", Facultad de Ingeniería, Grupos de investigación: \\ Ecitrónica y Geotecnia, Bogotá-Colombia. (e-mail: hernan.paz@escuelaing.edu.co; \\ sandra.campagnoli@escuelaing.edu.co; juan.gomez-ru@mail.escuelaing.edu.co; \\ juan.currea@mail.escuelaing.edu.co) \\ ${ }^{*}$ Autor a quien debe ser dirigida la correspondencia.
}

Recibido May. 17, 2018; Aceptado Jul. 24, 2018; Versión final Sep. 7, 2018, Publicado Abr. 2019

\begin{abstract}
Resumen
El objetivo de la investigación es la propuesta de un modelo teórico para caracterizar un sensor de fibra óptica. Para lograrlo, se caracterizó el cambio de las propiedades de los rayos de luz que se propagaban por el núcleo de la fibra, cuando el medio sufría un doblez proporcional a la deformación de un conjunto de cuatro muelles que soportaban el peso de un vehículo del tipo C2 (dos ejes). La deformación alteró el ángulo del doblez de la fibra de vidrio, que a su vez afectó la propagación de la luz debido a la presencia de ondas reflejadas y refractadas; este evento modificó la magnitud del coeficiente de transmisión (T) que se evaluó en el receptor. Este artículo presenta el estudio experimental -bajo condiciones controladas-, el registro, análisis de datos y regresión para modelar el sensor mediante una ecuación algebraica.
\end{abstract}

Palabras claves: sensor de fibra óptica; reflectometría de la luz; modelo teórico; sobrecarga vehicular

\section{Theoretical Model of Sensor based on the Principle of Light Reflectometry on Optical Fiber for Detection of Vehicular Overload}

\begin{abstract}
The objective of the research is to obtain a theoretical model that characterizes an optical fiber sensor. To achieve this, the change in the properties of the light rays that propagate through the core of the fiber was characterized, when the medium undergoes a fold proportional to the deformation of a set of four springs that supports the weight of a $\mathrm{C} 2$ vehicle (two axis). The deformation altered the angle of curvature ( $\alpha$ ) of the glass fiber, which in turn affected the propagation of light due to the presence of reflected and refracted waves. This event modified the magnitude of the transmission coefficient $(T)$ that was evaluated in the receiver. This article presents the experimental study -under controlled conditions-, the recording, data analysis and regression to model the sensor by means of an algebraic equation.
\end{abstract}

Keywords: optical fiber sensor; light reflectometry; theoretical model; vehicular overload 


\section{INTRODUCCIÓN}

El uso de sensores basados en fibra óptica se ha extendido desde su invención en el 2006 hasta la fecha (Wolfbeis y Bernhard, 2006). Los óptrodos se caracterizan por ser compactos (embebidos), no requieren de una señal de referencia, son inmunes a interferencia electromagnética y pueden ser interrogados remotamente. Buena parte de ellos, basan sus medidas en los cambios de fase, índice de refracción, polaridad de la onda, intensidad de luz (absorbida, emitida), tiempo de decaimiento de luminiscencia y espectros de vibración.

Los sensores basados en fibra óptica se pueden clasificar en puntuales o locales (interferómetros, microcurvaturas y polarización), cuasidistribuidos (redes de Bragg) y distribuidos (técnicas backscatter lineal: OTDR, OFDR; scattering Brillouin, scattering Raman); con estos sensores se puede monitorear la temperatura, espesor, distancia, tensión, esfuerzo, ángulo de rotación y vibración, salinidad, vibraciones, etc., en obras de ingeniería civil, mecánica, agrícola y biomédica; también en las industrias química, energética y bioquímica, y en la caracterización de materiales (Zhao et al., 2016). Para el caso que se ocupa este artículo, su enfoque es a la ingeniería Civil, para medir sobrecarga vehicular con un tipo de sensor basado en fibra óptica del tipo puntual. Específicamente, algunas de las aplicaciones de los sensores basados en fibra óptica en la ingeniería civil (vías asfálticas) se enfocaron en la medida de corriente polarimétrico (FBG: Fiber Bragg Grating y sensores totalmente distribuidos) para medir deformación longitudinal y microdeformación en losas (Kim et al., 2016; Chatti et al., 2008) con respuesta lineal y alta resolución. Otras usaron la reflectometría óptica (sensor distribuido lineal OTDR) para la detección de grietas (Childs et al., 2008; Bravo et al., 2012); también se utilizaron técnicas de modulación de la luz dispersada en inhomogeneidades aleatorias del índice de refracción del sensor para medir tensión en muestras de concreto (Chaube et al., 2008). Todas estas experiencias investigativas buscaban sensores más sensibles, estables, nuevas funcionalidades y fabricación a menor costo (Russo et al., 2011).

Para el caso de esta investigación se buscó modelar un sensor basado en fibra óptica extrínseco, del tipo reflectivo paralelo, para detección de sobrecarga de vehículos C2. Este tipo de vehículos de carga son camiones de dos ejes, sin articulación y con MMA mayor a 7,5 t.; representan el 52,6\% del parque automotor que circula en las carreteras de Colombia (Castellanos y Rojas, 2014). A pesar de regularse el peso máximo permitido de este vehículo en 17 t. (Ministerio de Transporte de Colombia, 2009), se excede en 10\% el peso máximo bruto permitido. Las sobrecargas además de causar daños considerables en los pavimentos dan lugar a mayores requerimientos para su mantenimiento, afectan la seguridad de circulación, y atentan contra la misma homologación de los vehículos en cuanto a que superan su capacidad. Adicionalmente, contaminan más el medio ambiente, ya que al desplazarse el C2 sobrecargado, emite al aire mayor cantidad de material particulado, polvo y humo. Actualmente, el tránsito de vehículos de carga en las vías colombianas, se controla mediante dos tipos de pesaje: estático y dinámico. A pesar del número suficiente de básculas en Colombia, la mayoría presentan problemas de calibración; también, se evidencia costos elevados en la operación y mantenimiento de las ya existentes, y para la adquisición de nuevos sistemas; en este contexto, se propone una nueva configuración de sensor de sobrecarga vehicular C2, más económico, funcional y confiable.

Se entendió por sensor un dispositivo capaz de detectar un cambio en un estímulo físico (variable de instrumentación) para transformarlo en una variable eléctrica; de igual manera, se interpretaron los términos "modelo teórico" como un paradigma conceptual mediante el cual se caracteriza y simplifica un fenómeno físico. Se buscó formular un modelo matemático numérico (ecuación, con variables y constantes) para el sensor basado en fibra óptica, que traduce la sobrecarga vehicular C2 en una medida del coeficiente de transmisión, como una relación entre las magnitudes de las señales recibida y transmitida del sistema. Para el desarrollo experimental de la investigación se partió de un tramo corto de fibra óptica multimodo, regular en la geometría del núcleo, homogénea y sin pérdidas; así mismo, se asumió una onda plana uniforme que se propagaba y confinaba en el núcleo en la dirección positiva de $z$. Sin embargo, el rayo podía alterar su trayectoria, si el tramo de fibra óptica sufría una curvatura.

En este caso, y como se muestra en la figura 1, la curvatura se ubicó en el eje $z=0$, y en ese punto se examinó el comportamiento de la luz como un modelo simplificado de rayos, mediante la ley de reflexión total (Yin et al., 2008). La onda plana uniforme puede incidir de dos maneras en el doblez: de modo normal y de modo oblicuo. Para el segundo caso, y como se observa en la figura 1, un rayo de luz en el medio 1 (nucleo) que incide con ángulo $\theta i$, una parte del rayo se refleja con ángulo $\theta r$ en direccion negativa de $z$ y la otra se refracta con ángulo $\theta$ t hacia el medio 2 (revestimiento). Las porciones del rayo de luz que se refleja y refracta, dependen de la reaccion de los dos materiales presentes en los dos medios frente al campo electrico E de la onda, y al ángulo de curvatura del doblez de la fibra óptica.

Puesto que la componente tangencial del campo eléctrico $E$ es continua en la frontera $Z=0$, la frecuencia no cambia y se acoplan las fases: $\theta r=\theta i$ y $\eta_{1} \operatorname{sen} \theta i=\eta_{2} \operatorname{sen} \theta_{t}$, que es la condición de reflexión total interna (ley 
de Snell). Con base en estas condiciones iniciales, se consideran dos casos: 1) El campo E es perpendicular al plano de incidencia, 2) El campo E es paralelo al plano de incidencia. Cualquier otra polarización representa una combinación lineal de estos dos casos. Los coeficientes de Fresnel de reflexión (ecuaciones 2 y 4 ) y de transmisión (ecuaciones 1 y 3), para una incidencia oblicua desde un medio sin pérdidas 1 a un medio sin pérdidas 2, para ambas polarizaciones, se muestran en la tabla 1.

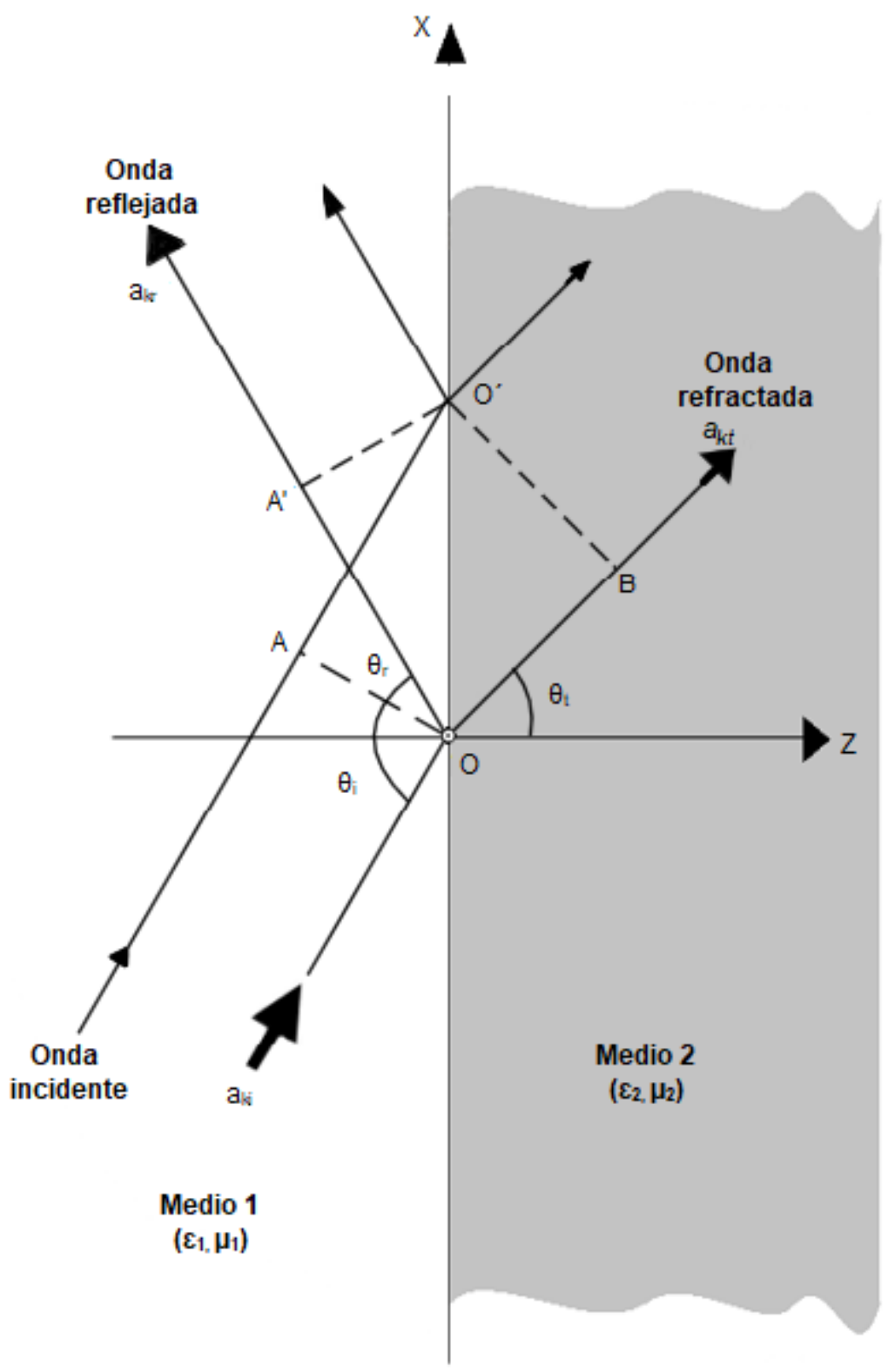

Fig. 1: Onda plana uniforme que incide oblicuamente sobre una frontera dieléctrica de fibra óptica curvada.

Tabla 1: Ecuaciones de los coeficientes de reflexión y transmisión para una onda plana uniforme con incidencia oblicua

\begin{tabular}{|c|ll|ll|}
\hline Tipo de polarización & \multicolumn{2}{|c|}{ Coeficiente de transmisión } & \multicolumn{2}{|c|}{ Coeficiente de reflexión } \\
\hline Polarización paralela & $\mathrm{T}=\frac{2 \eta_{2} \cos \theta_{i}}{\eta_{2} \cos \theta_{t}+\eta_{1} \cos \theta_{i}}$ & (1) & $\Gamma=\frac{\eta_{2} \cos \theta_{t}-\eta_{1} \cos \theta_{i}}{\eta_{2} \cos \theta_{t}+\eta_{1} \cos \theta_{i}}$ \\
\hline $\begin{array}{c}\text { Polarización } \\
\text { perpendicular }\end{array}$ & $\mathrm{T}=\frac{2 \eta_{2} \cos \theta_{\mathrm{i}}}{\eta_{2} \cos \theta_{\mathrm{i}}+\eta_{1} \cos \theta_{\mathrm{t}}}$ & (3) & $\Gamma=\frac{\eta_{2} \cos \theta_{\mathrm{i}}-\eta_{1} \cos \theta_{\mathrm{t}}}{\eta_{2} \cos \theta_{\mathrm{i}}+\eta_{1} \cos \theta_{\mathrm{t}}}$ \\
\hline
\end{tabular}

Para caracterizar el doblez de la fibra óptica se aplicaron conceptos de geometría euclidiana. El triángulo con base $y$, y altura $h$, cuenta con un ángulo $\alpha$ en el vértice. Este se evalúa como dos veces la función arcotangente de la relación $y / 2 h$ (ver figura 2). 


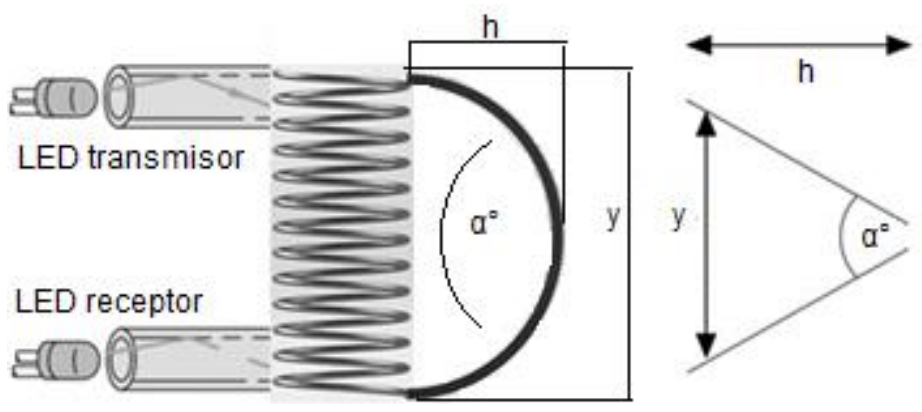

Fig. 2: Propagación del rayo de luz confinado en el núcleo de una fibra óptica, doblez de la fibra y alteración de la propagación del rayo de luz.

\section{METODOLOGÍA}

Se dividió en tres etapas: la primera consistió en pruebas de transmisión de señales no moduladas y moduladas a través del panel DL 2570 de fibra óptica. También se calibró el resorte, caracterizó el doblez de la fibra y se simuló en SolidWorks Student Edition®, el comportamiento intrínseco de la fibra óptica frente al esfuerzo. Los objetivos de esta etapa fueron seleccionar la señal de referencia -la de menor atenuación y variación de frecuencia en las pruebas-, calibrar el resorte, caracterizar el doblez y evaluar la capacidad de esfuerzo al cual se podía someter la fibra óptica.

La segunda etapa consistió en medir la carga vehicular mediante el coeficiente de transmisión de la luz ( $T$ ). Como se muestra en la figura 3, la fuerza ejercida por la máquina universal (que simula el peso del semieje direccional y no direccional del C2) deforma un resorte (muelle), sobre el cual se configura el doblez de un tramo de fibra óptica. Al transmitirse un tono modulado por intensidad de luz a través de la fibra óptica, una parte continua hacia el LED receptor, y otra parte se refleja y refracta en el doblez. Se mide la magnitud de la señal recibida y se divide sobre la magnitud de la señal transmitida (que no cambia) para sacar $T$ (ecuaciones 1 y 3). En la tercera y última etapa, se analizaron y promediaron los resultados de las pruebas, se construyeron curvas e hicieron regresiones para obtener el modelo teórico buscado. La construcción del sensor (a partir de la ecuación que lo modela) y su calibración serán los pasos a seguir en el futuro para la comprobación del correcto funcionamiento del mismo en la detección de sobrecarga vehicular.
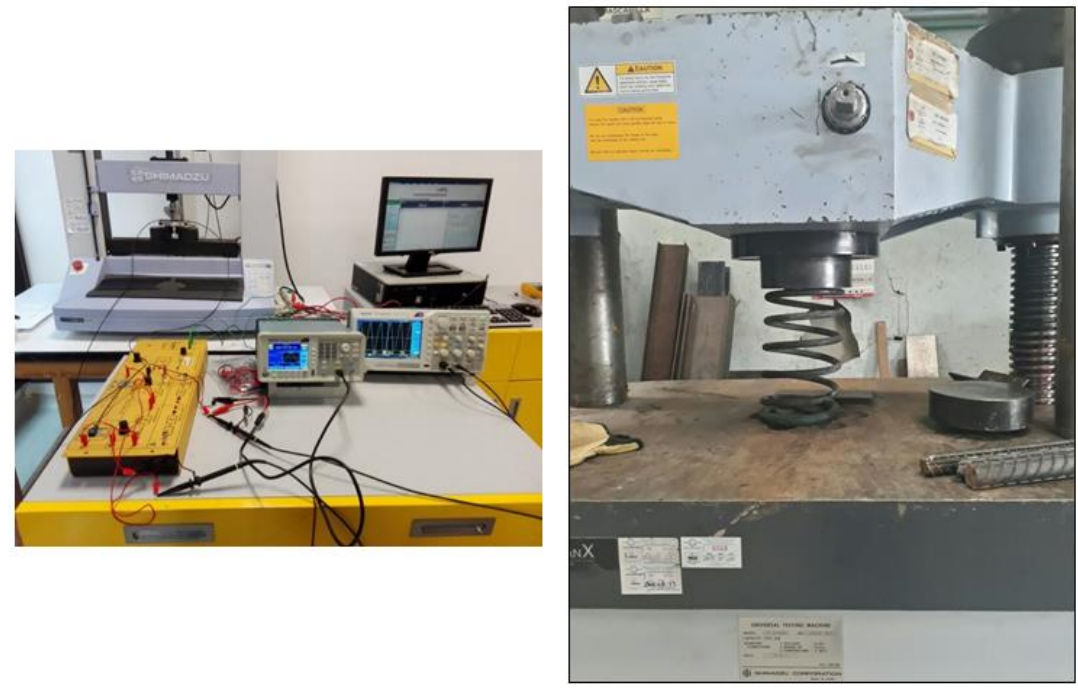

Fig. 3: Configuración del sistema sensor

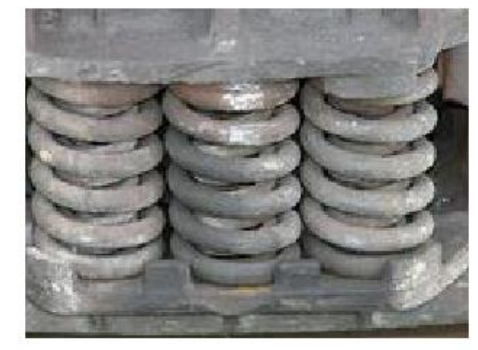


Los experimentos en el laboratorio se hicieron en condiciones controladas, siguiendo un protocolo y para una carga estática equivalente al vehículo C2. Este tipo de vehículo tiene un peso máximo de $17 \mathrm{t}$, distribuido en dos ejes simples: direccional de llanta simple (6 t) y no direccional de llanta simple para el camión pequeño, o de llanta doble para el camión grande (11 t). Como se muestra en la figura 4, el peso ejercido por el neumático con una presión de inflado de $5.92 \mathrm{~kg} / \mathrm{cm} 2$, sobre el área de contacto se representa de manera simplificada con una presión vertical de magnitud uniforme $W$ sobre un círculo de radio a $(a=10,5 \mathrm{~cm})$. Como la carga está uniformemente distribuida, se asume que los esfuerzos, deformaciones y deflexiones críticos ocurren en el eje de simetría bajo el centro del área circular (Rondón et al., 2014).

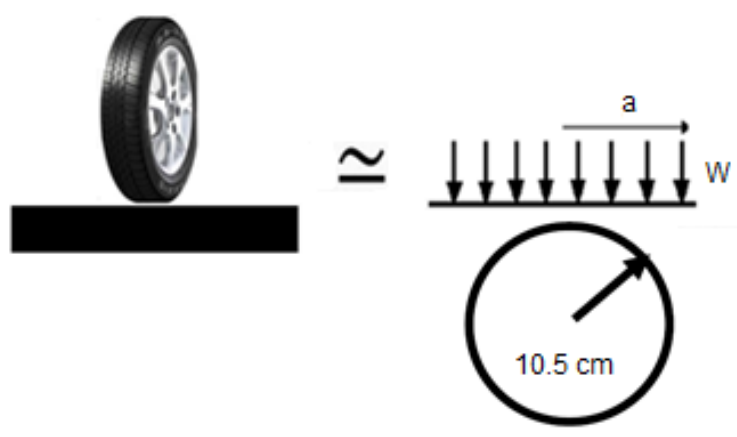

Fig. 4: Representación de la llanta como una placa de radio a y presión uniforme $W$

La superficie sobre la cual se hicieron los experimentos es un tapete con características equivalentes a las de un pavimento para un corredor de alto tráfico. Estas características especificadas en la tabla 2, toman como referencia a Herrera (2014) y fueron simuladas en el programa Weslea® (Tim, 2018).

Tabla 2: Características de pavimento para un corredor de alto tráfico localizado sobre la Autopista Norte entre las calles 134 y 128 de Bogotá.

\begin{tabular}{|l|l|l|l|}
\hline \multicolumn{1}{|c|}{ Capa asfáltica } & \multicolumn{1}{c|}{ Base granular } & \multicolumn{1}{c|}{ Subbase granular } & \multicolumn{1}{c|}{ subrasante } \\
\hline Módulo de elasticidad: & Módulo de elasticidad: & Módulo de elasticidad: & Módulo de elasticidad: \\
3464.8 MPa. & 206.8 MPa. & 103.4 MPa. & 25.8 MPa. \\
Coeficiente de capa: 0.30 & Coeficiente de capa: 0.14 & Coeficiente de capa: 0.11 & \\
Coeficiente de drenaje: 1 & Coeficiente de drenaje: 1 & Coeficiente de drenaje: 1 & \\
Espesor: $330.2 \mathrm{~mm}$. & Espesor: $152.4 \mathrm{~mm}$. & Espesor: $533.4 \mathrm{~mm}$. & \\
Relación de Poisson: 0.35 & Relación de Poisson: 0.45 & Relación de Poisson: 0.45 & \\
\hline
\end{tabular}

\section{RESULTADOS}

Como se muestra en la tabla 3, la transmisión de una señal no modulada de onda continua a través del panel DL 2570, con longitudes de fibra óptica de $5 \mathrm{~m}$ y $0.5 \mathrm{~m}$, es la que presenta menor atenuación y variación de frecuencia, mientras que las señales moduladas presentan atenuación y variación de frecuencia.

Tabla 3. Señales de tonos y moduladas en AM, FM, PM y FSK que se transmitieron y recibieron con el panel DL 2570. Nota: para la medición de las amplitudes, se usó una relación de 1 voltio por división en la escala vertical del equipo.

\begin{tabular}{|c|c|c|c|c|c|}
\hline \multirow[t]{2}{*}{ Modulación } & \multirow[t]{2}{*}{$\begin{array}{l}\text { Longitud de la } \\
\text { fibra óptica }\end{array}$} & \multicolumn{2}{|c|}{$\begin{array}{c}\text { Medidas de entrada (se usó una } \\
\text { relación de } 1 v \text { por división en la } \\
\text { escala vertical) }\end{array}$} & \multicolumn{2}{|c|}{$\begin{array}{l}\text { Medidas de salida (se usó una } \\
\text { relación de } 1 v \text { por división en la } \\
\text { escala vertical) }\end{array}$} \\
\hline & & $F(K H z)$ & Amplitud (Vpp) & $F(K H z)$ & Amplitud (Vpp) \\
\hline \multirow[t]{2}{*}{ AM } & $0.5 \mathrm{~m}$ & 200 & 0.472 & 200 & 0.472 \\
\hline & $5 m$ & 200 & 0.472 & 200 & 0.304 \\
\hline \multirow[t]{2}{*}{ FM } & $0.5 \mathrm{~m}$ & 69.44 & 0.422 & 67.55 & 0.422 \\
\hline & $5 m$ & 69.44 & 0.422 & 69.44 & 0.280 \\
\hline \multirow[t]{2}{*}{ PM } & $0.5 \mathrm{~m}$ & 50 & 1.24 & 60.98 & 1.24 \\
\hline & $5 m$ & 50 & 1.24 & 60.98 & 0.78 \\
\hline \multirow[t]{2}{*}{ FSK } & $0.5 \mathrm{~m}$ & 50 & 0.448 & 50 & 0.407 \\
\hline & $5 m$ & 50 & 0.448 & 50 & 0.245 \\
\hline \multirow[t]{2}{*}{ Tono sin modular } & $0.5 \mathrm{~m}$ & 2 & 0.210 & 2 & 0.210 \\
\hline & $5 m$ & 2 & 0.210 & 2 & 0.210 \\
\hline
\end{tabular}


En la simulación en SolidWorks de los esfuerzos debidos a una carga distribuida $\mathrm{W}=3 \mathrm{t}$., a los que estuvo expuesto el cable de fibra de $0.5 \mathrm{~m}$, se presentaron desplazamientos transversales y longitudinales máximos del orden de $53.14 \mu \mathrm{m}$ y $2.81 \mu \mathrm{m}$ respectivamente. Estas deformaciones fueron proporcionales a la distancia del punto de aplicación de la fuerza.

La calibración del resorte (muelle) y la caracterización del doblez de la fibra óptica que se realiza en esta investigación es en realidad una caracterización de su respuesta, pero en ningún momento se pretende realizar calibraciones rigurosas en términos de patrones homologados. Con esta perspectiva, se determinó la constante elástica del resorte $\mathrm{K}$ (muelle) mediante la ley de Hooke; para ello, se hicieron pruebas de laboratorio para medir la compresión de un muelle cuando era sometido a una carga (fuerza) en un intervalo de $0 \mathrm{~N}$ a $811 \mathrm{~N}$ (análisis de la curva de carga en condiciones estáticas); posteriormente se aplicó la ley de Hooke: $\mathrm{F}=-\mathrm{K} \Delta \mathrm{x}$. Teóricamente los puntos obtenidos debían estar sobre una recta de pendiente $\mathrm{K}$ según predice la ley de Hooke, pero los errores experimentales hicieron que quedaran fuera de ella. En este escenario, se halló una recta cuyos puntos se separasen lo menos posible de todos los hallados y que quedaran lo más equidistante posible a todos ellos (Matar et al., 2017). Una vez hallada la recta se eligieron dos puntos bastante separados para hallar su pendiente, que condujo a la figura 5 . La constante elástica del resorte (muelle) fue de $\mathrm{K}=15,955 \mathrm{Kg} / \mathrm{mm}$, que expresada en $\mathrm{N} / \mathrm{mm}$, dio: $K=15,955 \mathrm{Kg} / \mathrm{mm}$ * $9.81 \mathrm{~m} / \mathrm{s}=156,52$ $\mathrm{N} / \mathrm{mm}$.

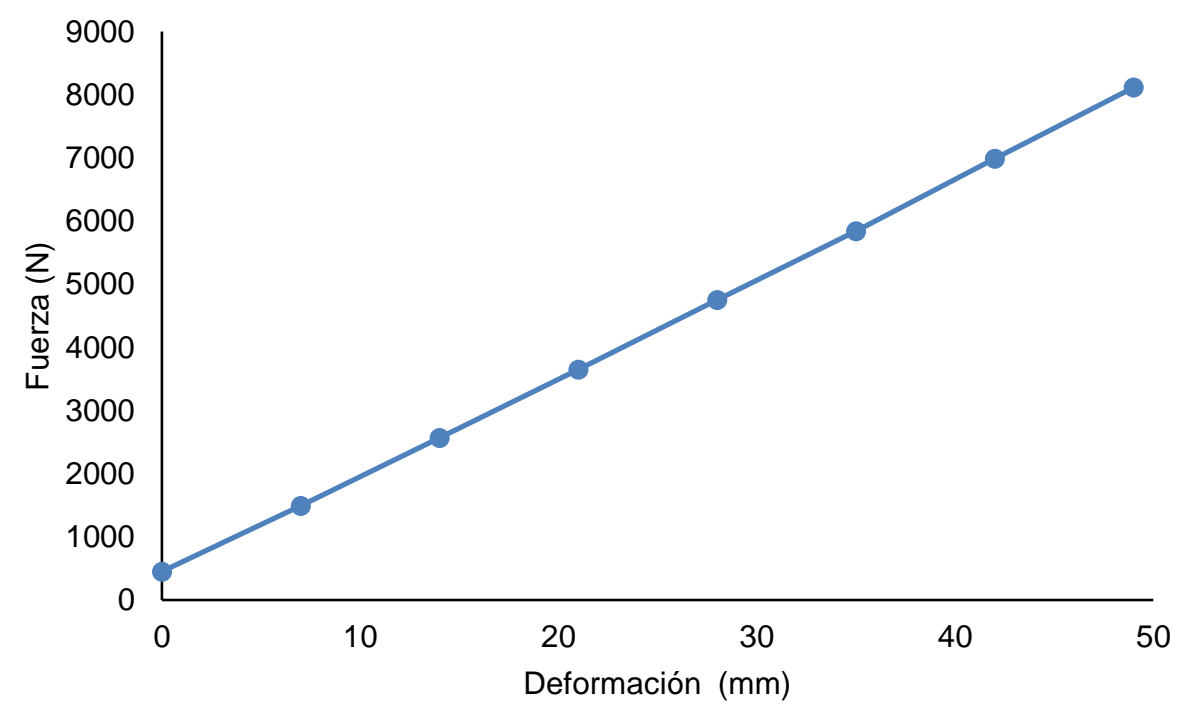

Fig. 5: Regresión para hallar la constante elástica del muelle K.

La ecuación que relaciona la deformación del resorte $\Delta x$ con el ángulo $\alpha$, fue: $\Delta x=y-2 h \tan (\alpha / 2)$, donde $y$ es la altura del resorte y $h$ es la distancia del arco (ver figura 2). Despejando el ángulo de la expresión $\Delta x$ se obtuvo la ecuación 5.

$$
\alpha=2 \operatorname{arcotan}\left(\frac{\mathrm{y}-\Delta \mathrm{x}}{2 \mathrm{~h}}\right)
$$

La ecuación que relacionó la fuerza ejercida por el vehículo C2 sobre el resorte con el ángulo del doblez de la fibra óptica, fue la expresión $F=-K \Delta x$, donde $K$ era la constante elástica del muelle. Al reemplazar $\Delta x$ en $F$, se obtuvo: $\mathrm{F}=\mathrm{K}[\mathrm{y}-2 \mathrm{htan}(\alpha / 2)]$. Los valores iniciales del sistema sensor, antes que fuera sometido a sobrecarga, fueron de: $y=365 \mathrm{~mm}$ y $h=150 \mathrm{~mm}$. Sustituidos estos valores en $F$, se obtuvo la ecuación 6 .

$$
\mathrm{F}=156,52\left(365-2(150) \tan \left(\frac{\alpha}{2}\right)\right)
$$

Debido a las grandes magnitudes de sobrecarga que debía soportar el muelle (hasta $11 \mathrm{t}$ en el semieje no direccional), que fácilmente lo podía deformar más allá de su punto crítico (punto en el cual se ponen en contacto dos espiras adyacentes), se optó por seleccionar 4 muelles idénticos y agruparlos en un conjunto. Con este arreglo, la sobrecarga se distribuyó de manera proporcional y no se superó la limitación mencionada arriba. El conjunto se configuró para cubrir el área del neumático del vehículo C2 (ver figura 3). Las tres pruebas del sistema sensor (panel DL 2570, doblez de la fibra, máquina universal y el conjunto de muelles) arrojaron resultados de variables, que se promediaron, estas fueron: $\Delta \mathrm{Y}_{\text {pro }}(\mathrm{mm})$, representa la deformación promedio del resorte, cuando es sometido a distintas cargas (que también se promediaron Cargapro); el voltaje resultante y promediado $\left(\mathrm{V}_{\text {pro }}\right)$, medido con un multímetro, a la salida del panel permitió hallar el coeficiente 
de trasmisión promedio ( $\left.\mathrm{T}_{\text {pro }}\right)$ de las mismas pruebas, que se tabulan en la tabla 4 . Con los datos de la tabla 4, se trazó la curva característica $T$ vs $F$, de la figura 6 . $F_{\text {pro }}(\mathrm{N})$

Tabla 4: Resultados promedio de tres pruebas de laboratorio.

\begin{tabular}{|c|c|c|c|c|c|}
\hline$\Delta Y_{\text {pro }}(\mathrm{mm})$ & $V_{\text {pro }}\left(m V_{R M S}\right)$ & Cargapro $(\mathrm{kg})$ & $F_{p r o}(N)$ & $K(\mathrm{~N} / \mathrm{mm})$ & $T_{\text {pro }}$ \\
\hline 0 & 344,88 & 46 & 451,26 & - & 1,0000 \\
\hline 7 & 337,46 & 152 & 1491,12 & 147,15 & 0,9785 \\
\hline 14 & 330,73 & 261,5 & 2565,32 & 152,055 & 0,9590 \\
\hline 21 & 324,39 & 372 & 3649,32 & 152,055 & 0,9406 \\
\hline 28 & 315,48 & 484,5 & 4752,95 & 161,865 & 0,9148 \\
\hline 35 & 304,68 & 595 & 5836,95 & 147,15 & 0,8834 \\
\hline 42 & 289,76 & 712 & 6984,72 & 166,77 & 0,8402 \\
\hline 49 & 275,38 & 827 & 8112,87 & 156,96 & 0,7985 \\
\hline
\end{tabular}

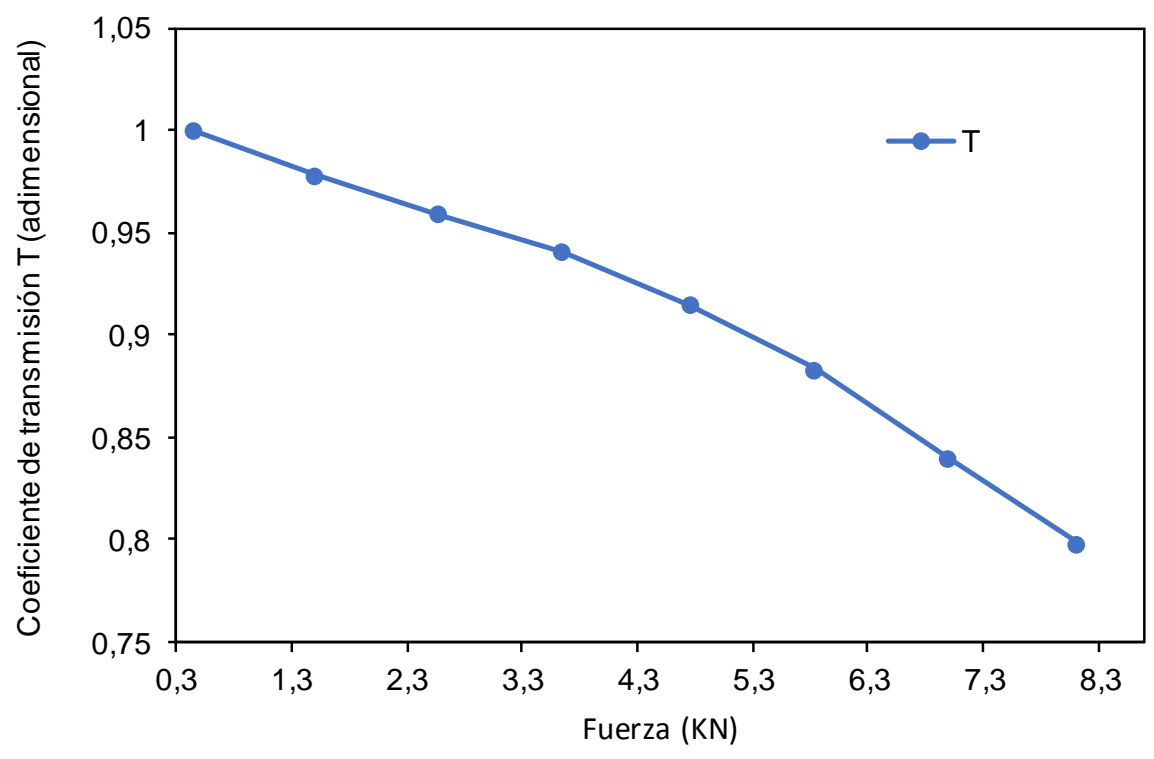

Fig. 6: Curva característica de T vs F

Teniendo en cuenta el comportamiento de los datos mostrados en la figura 6 , se optó por una regresión no lineal a una función polinomial. Para este caso de estudio, la regresión polinomial se ajustó a tres términos de la variable independiente: $y=a+b x+c x^{2}$. Sí se deriva esta ecuación con respecto a sus coeficientes, se obtiene un sistema de ecuaciones $\mathrm{S}_{\mathrm{am}}$, donde $m$ simboliza el número de pares de datos. Para el sistema en cuestión se necesitó $m=8$, debido a los ocho términos registrados del coeficiente de transmisión (columna 6, tabla 3). Posteriormente se hallaron los términos para la solución del sistema de segundo grado; las magnitudes de estos términos se obtuvieron mediante operaciones algebraicas y se muestran en la tabla 5.

Tabla 5: Valores de las incógnitas

\begin{tabular}{|c|c|c|c|c|}
\hline$a$ & $b$ & $c$ & $y$ \\
\hline 8 & 33844,5 & 193590896,5445 & $=$ & 7,3149 \\
\hline 33844,5 & 193590896,5445 & 1249862296861,33 & $=$ & 29653,587 \\
\hline $\begin{array}{c}193590896,544 \\
5\end{array}$ & 1249862296861,33 & 8608943697391320 & $=$ & 165523843,4083 \\
\hline
\end{tabular}

La ecuación final que modela el sistema sensor basado en fibra óptica, es de la forma: T=1,001-0,00001F$1,8 \times 10^{-7} F^{2}$. Las raíces de la ecuación cuadrática son: $x=-26522,8$ y $x=20967,3$. La figura 6 muestra la curva del coeficiente de transmisión $T$ con respecto a la fuerza ejercida por la máquina universal (que simula el peso del semieje direccional o no direccional del vehículo $C 2$ ). La fuerza $F$ a la que hace referencia la ecuación anterior, debe considerar la fuerza de oposición de todos y cada uno de los cuatro muelles $\left(F_{n}\right)$ que conforman 
el conjunto $(F)$, ya que la fuerza que relaciona la deformación del sistema sensor está acotada por la constante de rigidez axial. Al considerar los 4 muelles en el conjunto, se toma en cuenta un factor multiplicativo de $F$ por 4 en la ecuación de $T$. Finalmente el sensor basado en el principio de reflectometría de la luz sobre fibra óptica queda modelado con la ecuación 7:

$$
\mathrm{T}=1,001-0,00001 \frac{F_{n}}{4}-1,8 \times 10^{-9} \frac{F_{n}^{2}}{4}
$$

\section{CONCLUSIONES}

Se muestra el desarrollo experimental, junto con el análisis de resultados para obtener el modelamiento teórico del sensor mediante una ecuación polinomial de segundo grado. La detección de sobrecarga vehicular C2, de manera indirecta (mediante desplazamiento del conjunto de muelles, que afecta el doblez de la fibra óptica) y a través de la variación del coeficiente de transmisión $T$, que depende del doblez de la fibra óptica es novedoso en el ámbito científico. Sin embargo, la sensibilidad del sensor se podría comprometer debido a las pérdidas en el dieléctrico debido al doblez, y que pueden variar de acuerdo al tipo de fibra óptica y dimensiones transversales de la misma. Se halló que el tono era la señal más adecuada para hacer los experimentos de laboratorio. Los pesos generados por el semieje direccional simple (3t) y no direccional de llanta doble (5.5 t) del vehículo $\mathrm{C} 2$ y la temperatura en las que fueron hechas las pruebas, no superan los límites de deformación crítica del conjunto de muelles, tampoco superan los límites de elasticidad y temperatura de los materiales que componen la fibra $\left(2410 \mathrm{MPa}\right.$ y $\left.70^{\circ} \mathrm{C}\right)$, por lo que el prototipo utilizado para la aproximación al modelo teórico del sensor cumplía con los requisitos de carga y temperatura. Como trabajo futuro se tiene previsto validar el modelo teórico desarrollado, con ensayos sobre cargas calibradas (carga actuante y tipo de vehículo). Estos ensayos permitirían asignarle a la ecuación un factor de corrección para compensar su deriva y lograr su bondad; posteriormente, construir el sensor; y por último, validar la funcionalidad del mismo.

\section{REFERENCIAS}

Castellanos, A.P. y J.P. Rojas, Espectros de carga vehicular para la zona urbana de Bogotá D.C., Universidad de Los Andes (2014)

Chatti, K., A. Manik y N. Brake, Effect of axle configurations on fatigue and faulting of concrete pavements, $10^{\text {th }}$ International Symposium on Heavy Vehicle Transport Technology, Paris, Francia, 117-126 (2008)

Chaube, P., B.G. Colpitts, D. Jagannathan y A.W. Brown, Distributed FiberOptic Sensor for Dynamic Strain Measurement, ISSN: 1530-437X, IEEE Sensors Journal, 8(7), 1067-1072 (2008)

Childs, P., A.C. Wong, W. Terry y G.D. Peng, Measurement of crack formation in concrete using embedded optical fibre sensors and differential strain analysis, ISSN: 0957-0233, Measurement science and technology, 19(6), 065301 (2008)

Herrera, I.C., Diseño del pavimento para un corredor de alto tráfico localizado sobre la Autopista Norte entre las calles 134 y 128, Bogotá (2014)

Kim, T.M., D.H. Kim, M.K. Kim e Y.M. Lim, Fiber Bragg grating-based long-gauge fiber optic sensor for monitoring of a $60 \mathrm{~m}$ full-scale prestressed concrete girder during lifting and loading, doi:10.1016/j.sna.2016.10.037, Sensors and Actuators, A: Physical, 252, 134-145 (2016)

Matar, M., M. Parodi, C. Repetto y A. Roatta, Modelización lineal de un sistema masa-resorte real, doi: http://dx.doi.org/10.1590/1806-9126-RBEF-2017-0238, Revista Brasileira de Ensino de Física, 40, 2, e2306 (2018)

Ministerio de Transporte de Colombia, Resolución Nº 001782 (2009)

Rondón, H., E. Delgadillo y W. Vargas, Diseño, construcción y funcionamiento de un prototipo para medir cargas vehiculares en un pavimento flexible, Revista Ingeniería de Construcción, 29(1) (2014)

Russo, N.A., S.B. Noriega y R. Duchowicz, Implementación de Sistema Óptico para Grabado de Redes de Bragg en Fibra Óptica. http://dx.doi.org/10.4067/S0718-07642011000200013, Información Tecnológica, 22(2), 121-130 (2011)

Tim, D.H. Weslea®, Department of Civil Engineering, Auburn University, EEUU, (2010)

Wolfbeis, S. y M.W. Bernhard, Fiber optic chemical sensors and biosensors. A view back, in optical chemical sensors, Sringer, 2, 95-111 (2006)

Yin, S., P.B. Ruffin y F.T. Yu, Fiber Optic Sensors, $2^{\text {nd }}$ Ed., ISBN: 978-1-4200-5365-4, CRC Press Book, New York, USA (2008)

Zhao, Y., X. Li, L. Cai e Y. Zhang, Measurement of RI and temperature using composite interferometer with hollow-core fiber and photonic crystal fiber, doi:10.1109/TIM.2016.2584390, IEEE Transactions on Instrumentation and Measurement, 65(11), 2631-2636 (2016) 